\title{
A Stenciling Delay System for Steel Plate Ultrasonic Flaw Detection Process \\ Gengzhu Wang
}

University of Science and Technology Liaoning, Anshan, Liaoning, 114051, China

wgz20203@sina.com

\section{Key Words: Stenciling delay system, Detection, Software}

\begin{abstract}
In the process of steel plate ultrasonic flaw detection, once the instrumentation discovers the flaw, a sign is stenciled in the vertical direction of the flaw, because there is a distance between the detector and stenciling, an effective method must be applied to reasonably control the stenciling, in order to achieve the precise stenciling. Based on characteristics of the steel plate flaw detection process, the delay distance between each group of detectors and stenciling has been analyzed, simultaneously based on the flaw detection speed, stenciling delay data has obtained. Stenciling delay structure is developed using the computer software technique, instead of hardware control mode, software algorithm is carried out to design, which has achieved the goal of convenience application and precise orientation.
\end{abstract}

\section{Introduction}

When the instrument detects the signal, how to automatically mark at the place of the defect is one of the important issues of automatic flaw detection. During the horizontal movement of the sector-led probe and stenciling device on the gantry, once the defect is found, the detection system will notify the spray system to operate ${ }^{[1]}$. Because of the mechanical structure characteristics of the ultrasonic detection device (Figure 1), the spray gun is normally mounted at the rear of the probe holder, which can avoid complex mechanical structure. And the automated flaw detection often uses water as coupling medium, when using spray paint to mark, it is apparently can not be realized at the places with water, only when the defect is moved away from the region of water, it can be stenciled at the defect. In the straight seam tube automatic flaw detection, normally eight channels are used to detect the flaw in its horizontal and vertical directions ${ }^{[2]}$. Therefore, the results of detection for each channel must be accurately labeled, in order to carry out effective treatment. In terms of this situation, it can be achieved by hardware and software method respectively.

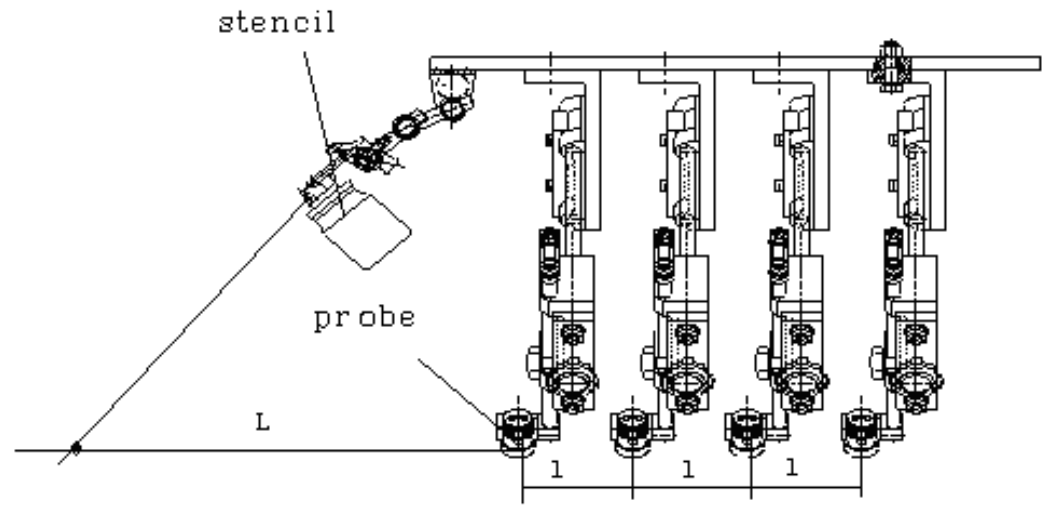

Fig.1 The mechanical construction of the probe and the stenciling equipment 


\section{The hardware system analysis of the stenciling control}

To solve the above problems, some automatic flaw detector has the delay braking circuits that can delay 0 to 10 seconds. The circuit consists of several or dozens of mono stable circuits, the pulse width and the inter-relationship of the stable circuits are shown in Figure 2. The minimum delay time is 0.2 seconds, after an alarm signal went through the delay brake circuit, which is up to 9.8 to 10.1 seconds. If the two pulse outputs are required from the delay brake circuits, you must make the time interval between two adjacent pulses is greater than the pulse width $t_{l}$ plus the recovery time $t_{H}$ of the first mono stable circuit. Because the resolution of the delay brake circuit is greater than $t_{1}+t_{H}$, which means for signal with pulse interval is greater than $t_{1}+t_{H}$, delay brake circuit has save function.

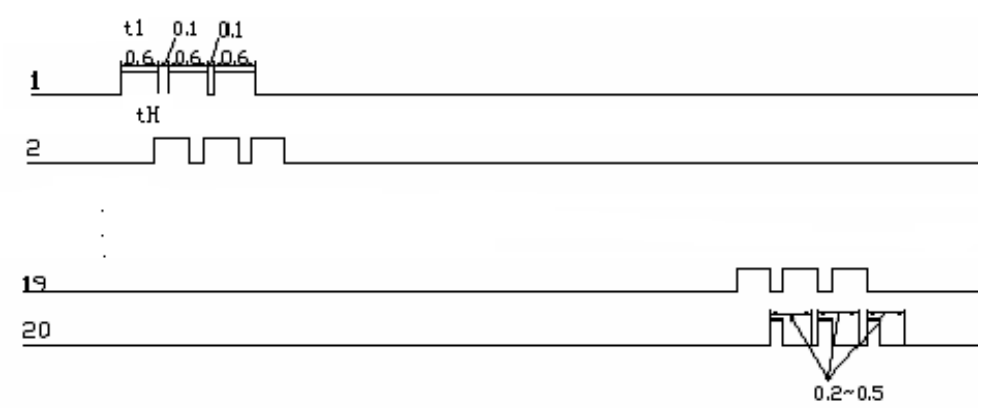

Fig.2 The pulse width and time relationship of the each stable circuit

Since the delay brake circuit has the function of saving the defect signals, when a defect is discovered, if the work piece needs to be inspected over and over, the defect signal stored in the circuits must be all eliminated to avoid the false stenciling during the inspection ${ }^{[3]}$. To avoid this happening, when the work piece is moving backwards or stopping, the transmission device should send out a signal to the brake circuits to clear the signal stored in the original storage. To meet the above requirements, the delay brake circuit must have the following features: that is, it should have a relatively short recovery time, and after the trigger it can be forced to restore the initial state. From the above analysis it can be seen that the stenciling system hardware control mode is characterized by

(1) The hardware control circuit structure of the stenciling system is relatively complicated, but the processing is speedy, and no computer processing time is used.

(2) It is more affordable, but it is less applicable. Once the detecting conditions changed, especially it is difficult to adapt to detecting different diameter pipes.

(3) It is more cumbersome to adjust. Since each channel has different stenciling delay time, each of them must be individually adjust to achieve the overall control.

For digital automatic flaw detection, computer is widely used as a means of signal processing. By this effective method, simply adding a very small portion of the interface circuits, using software approach to simulate its hardware control, the effect will be better.

\section{Software system analysis of the stenciling control}

The longitudinal welded pipe ultrasonic flaw detector consists of probe set, spray gun, crane, roller, and gantry etc. The longitudinal welded pipe is held up by the roller and rotates to align with the weld seam. The detector and the spray gun are uniformly installed on the crane, through the operation of the crane to test the entire pipe. In the testing process, if a flaw at a place is detected by the probe, the probe will mark at the place ${ }^{[4]}$. 
According to the mechanical structure shown in Figure 1, the distance between the probes is $1=$ $50 \mathrm{~mm}$ (based on the probe scanning ranges, in the practical application $\mathrm{s}=25 \mathrm{~mm}$, the front and rear probes are arranged in pairs), the distance between the probe and the spray is $\mathrm{L}=260 \mathrm{~mm}$ (measured at the spot), then the distance between the channel and spray are respectively $285,310,335,360,385,410,435,460 \mathrm{~mm}$. In actual testing, because the repetition frequency is $500 \mathrm{~Hz}$, the one time scanning is $2 \mathrm{~ms}$. According to testing standards, for a $\phi 3$ hole testing, the same probe has to carry out three consecutive defects (software flaw detection requirements), thus requiring the maximum linear speed of detection is

$$
v_{\max }=\frac{d}{n t}
$$

Where, $d$ is the diameter of the detected standard hole. $n$ is the detection times. $t$ is repeat time.

Then it is known that $v_{\max }=30 \mathrm{~m} / \mathrm{min}$, in the actual application $v_{\max }=24 \mathrm{~m} / \mathrm{min}$. Therefore for a $\phi 219$ seamless pipe, its horizontal maximum speed is obtained.

$$
v_{-\max }=\frac{L v_{\max }}{\pi D}
$$

Where, $D$ is the diameter of the seamless steel tube. $L$ is the pitch.

The probe set consists of eight probes. The instrument synchronization circuit sends out $4 \mathrm{KHz}$ synchronization pulse, that is, the pulse frequency is $\mathrm{f}=4 \mathrm{KHz}$. Each pulse time is $250 \mu \mathrm{s}$, so each probe sends out one time of ultrasound at interval of $250 \mu \mathrm{s}$. One cycle of run for 8 probes needs $2 \mathrm{~ms}$, then the repetition frequency is $500 \mathrm{~Hz}$. Therefore, the detection pitch is $\mathrm{L}=25 \times 4=100 \mathrm{~mm}$ (in general, according to the actual detecting requirements, horizontal and vertical wound flaw, each occupies 4-channel), so $v_{\max }=3.5 \mathrm{~m} / \mathrm{min}$. It can be seen, after one detection cycle, the maximum travel distance of the run is $0.115 \mathrm{~mm}$. If the time accuracy is $0.1 \mathrm{~s}$, the maximum limit of the spray position control accuracy is $0.115 \times 0.1 / 2 \times 1000=5.75 \mathrm{~mm}$. For regular users the required precision control is about $10 \mathrm{~mm}$, which can be fully realized.

From the above analysis, the software-controlled methods are: first the host computer gives the delay time based on the detection speed. Assumed that the first channel needs 20s delay time, and the accuracy is $0.1 \mathrm{~s}$, to make the delay time [0] $=200$ through the communication, (by delay time [0] $=$ Read Control. Read Char [13] statement to obtain). So the array delay time [0] size is 200 to ensure all of the delay process, ie pen [0]. The maximum value of Delay Control is 199, each element interval of the array delay time [0] is $0.1 \mathrm{~s}$. If there is no flaw, the element is in low level, that is, pen [0]. Delay Data [pen [0]. Delay Control] == 0; if injury element is high, that is, pen [0]. Delay Data [pen [0] . delay Control] $==1$.

During the stenciling action, it needs to determine if the next element of the current array element is 1. Assuming the current is pen [0]. Delay Control $=5$ there has flaw, that is pen [0]. Delay Data [pen [0]. Delay Control] $==1$, the program circularly determines if the pen [0]. Delay Data [pen [0]. Delay Control +1 ] is 1 til1 the pen [1]. Delay Control to maximum, ie 199, then doing spray, the detailed flow chart 2 is shown in Fig. ${ }^{[5]}$.

\section{Summary}

Comparing to the hardware control, the advantages of the software control of the stenciling control system are

(1) simple structure, easy to control

(2) easy to modify the control circuit

(3) wider range of the spray delay time settings

(4) Easy to apply and wide adaptability. In the process of detection of different diameters of steel pipes, simply enter the parameters; the computer can directly conduct the detection. 


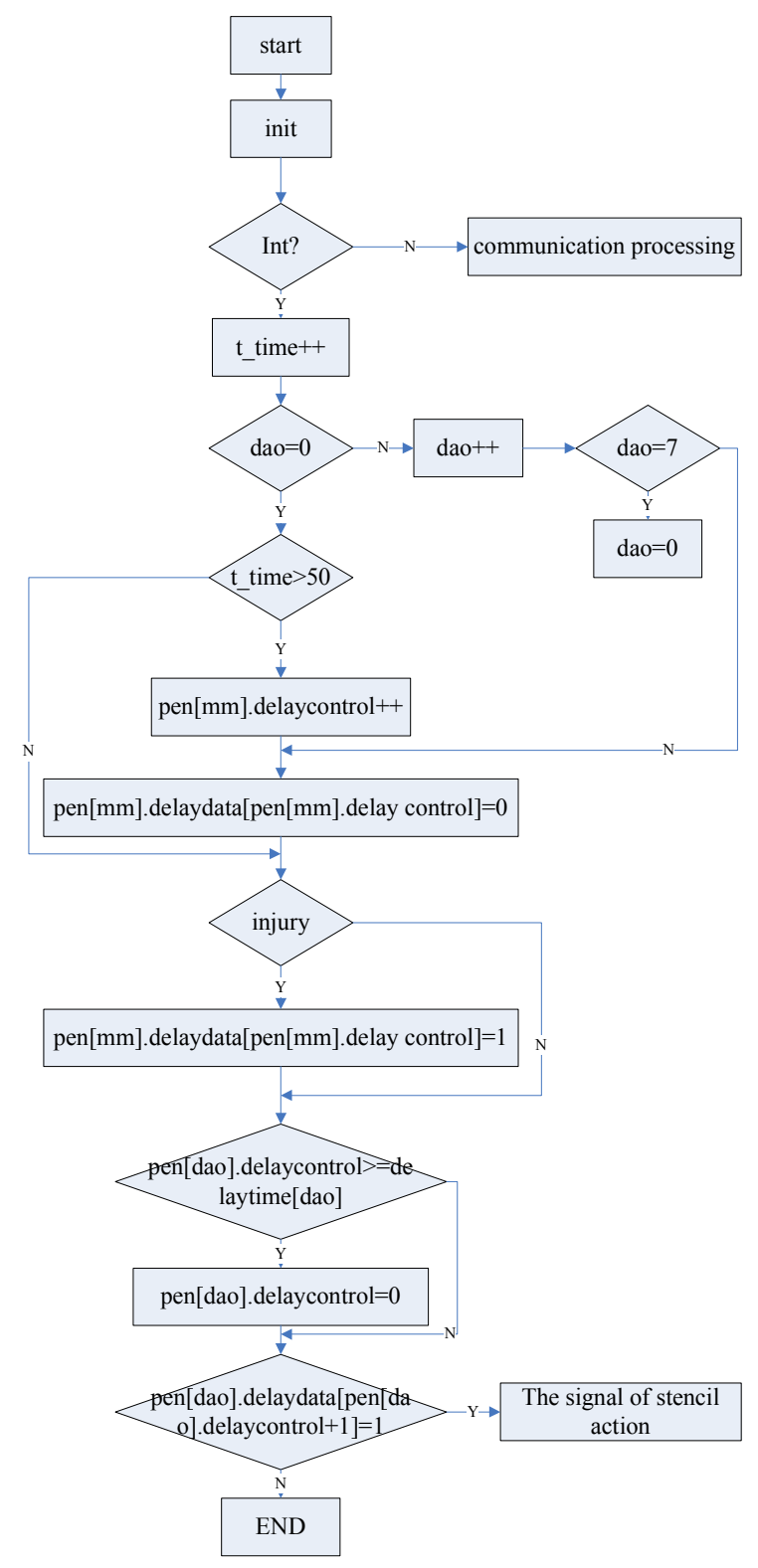

Fig.3 The program flow chart

\section{References}

[1] Kazys, R. Analysis of application for ultrasonic testing[J], Ultrasonics, 1995

[2] R. J. Ditchburn, S . K. Burke and C. M. Scala, NDT of welds: state of the art[J]. NDT\&E International.1996:111-115.

[3] Ge Xin, Wu Xiaomei, Wang Yuanyuan, Fang Zuxiang. Research Progress in Electromagnetic Tracking Method Chinese Journal of Medical Instrumentation2011.2:119-122

[4] F. Raab,E. Blood,T. Steioner,H. Jones. Magnetic position and orientation tracking system .IEEE Transactions on Aerospace and Electronic Systems, 1979,15, 15 (5) :709-718 .

[5] Bruce Eckel. Thinking in C++(Second Edition). Prentice Hall ,2002.1 